\title{
Thermal Parameters in Heavy Ion Collisions at SPS and RHIC: Centrality Dependence
}

\author{
B. KämpfeR ${ }^{a}$, J. Cleymans ${ }^{b}$, K. Gallmeister ${ }^{a, c}$, S.M. Wheaton $^{b}$ \\ ${ }^{a}$ Forschungszentrum Rossendorf, PF 510119, 01314 Dresden, Germany \\ ${ }^{b}$ University of Cape Town, Rondebosch 7701, Cape Town, South Africa \\ ${ }^{c}$ Institut für Theoretische Physik, Universität Giessen, Giessen, Germany*
}

\begin{abstract}
The centrality dependence of thermal parameters describing hadron multiplicities and intermediate-mass dilepton spectra in heavy-ion collisions at SPS and RHIC is analyzed. From experimental hadron multiplicities we deduce evidence for strangeness saturation at high energy and maximum centrality. The observed dilepton spectra can be parameterized by a centrality independent temperature.
\end{abstract}

keywords: relativistic heavy-ion collisions, thermal models, hadron yields, dileptons PACS: 24.10.Pa, 25.75.Dw, 25.75.-q, 27.75.+p, 12.38.Mh, 24.85.+p

\section{INTRODUCTION}

It has been shown that various observables of relativistic heavy-ion collisions can be well described by statisticalthermal or hydrodynamical models. In such a way, a selected subset of a large number of observables can be reproduced by a small number of characteristic parameters, such as temperature, density or flow velocity. It is the subject of the present note to pursue this idea and to analyze the centrality dependence of the thermal parameters describing hadron multiplicities and dilepton spectra in the intermediate-mass region. This will provide further information about the effects of the size of the excited strongly interacting system and help in the systematic understanding of the experimental data.

The aim of the present work is twofold: (i) the previous analysis [1] of hadron multiplicities at SPS and RHIC is repeated and improved, e.g., by including feed-down from weak decays, and (ii) the consideration of the intermediatemass dilepton spectra at SPS is outlined in more detail, in particular by analyzing both the invariant mass and transverse momentum spectra.

\section{HADRON MULTIPLICITIES}

Hadron multiplicities can be reproduced [2, 3] by the grand-canonical partition function $\mathcal{Z}_{i}\left(V, T, \mu^{\alpha}\right)=$ $\operatorname{Tr}\left[\exp \left\{-\left(\hat{H}-\mu^{\alpha} Q_{i}^{\alpha}\right) / T\right\}\right]$, where $\hat{H}$ is the statistical operator of the system, $T$ denotes the temperature, and $\mu^{\alpha}$ and $Q_{i}^{\alpha}$ represent the chemical potentials and corresponding conserved charges respectively. In the analysis of $4 \pi$ data, the net-zero strangeness and the baryon-to-electric charge ratio of the colliding nuclei constrain the components $\mu^{\alpha}=\left(\mu_{B}, \mu_{S}, \mu_{Q}\right)$, where the subscripts $B, S$ and $Q$ refer to baryon, strangeness, electric charge. These constraints have to be relaxed when considering data in a limited rapidity window, increasing the number of free parameters. The particle numbers are given by

$$
N_{i}^{\text {prim }}=V\left(2 J_{i}+1\right) \int \frac{d^{3} p}{(2 \pi)^{3}} d m_{i}\left[\gamma_{s}^{-S_{i}} \mathrm{e}^{\frac{E_{i}-\mu^{\alpha} Q_{i}^{\alpha}}{T}} \pm 1\right]^{-1} \mathrm{BW}\left(m_{i}\right),
$$

where we include phenomenologically a strangeness saturation factor $\gamma_{s}$ (with $S_{i}$ the total number of strange quarks in hadron species $i$ ) to account for incomplete equilibration in this sector, $E_{i}=\sqrt{\vec{p}^{2}+m_{i}^{2}}$, and BW is the BreitWigner distribution (to be replaced by a $\delta$-function for stable hadrons). The final particle numbers are $N_{i}=N_{i}^{\text {prim }}+$ $\sum_{j} \mathrm{Br}^{j \rightarrow i} N_{j}^{\text {prim }}$ due to decays of unstable particles with branching ratios $\mathrm{Br}^{j \rightarrow i}$. Such a description can certainly be justified for multiplicities measured over the whole phase-space, since many dynamical effects cancel out in ratios of hadron yields.

* present address 
We have analyzed two data sets: (i) NA49 $4 \pi$ multiplicities of $\langle\pi\rangle=\frac{1}{2}\left(\pi^{+}+\pi^{-}\right), K^{ \pm}, \bar{p}, \phi$, and $N_{\text {part }}$ (taken as the sum over all baryons) in 6 centrality bins in the reaction $\mathrm{Pb}(158 \mathrm{AGeV})+\mathrm{Pb}$ 《], 5] (it should be emphasized that protons are not included in our analysis [6] since there may be a spectator component in non-central collisions), and (ii) PHENIX mid-rapidity densities of $\pi^{ \pm}, K^{ \pm}$, and $p^{ \pm}$in the reaction $\mathrm{Au}+\mathrm{Au}$ at $\sqrt{s}=130 \mathrm{AGeV}$ in 5 centrality bins [7]. In [7] it is estimated that the probability for reconstructing protons from $\Lambda$ decays as prompt protons is $32 \%$ at $p_{T}=1 \mathrm{GeV} / \mathrm{c}$. This is the probability for feed-down used in our present fits to the mid-rapidity PHENIX data. The results of our fits are displayed in Fig. 1. Specific features of the $\phi$ mesons at SPS will be discussed elsewhere [8].

A comparison of the individual thermal parameters of both data sets is displayed in Fig. 2, which corrects Fig. 2 in [1] (the correction of a coding error in [1] lowers the temperature and brings it in agreement with [3]). Most remarkable is the drop of the baryo-chemical potential $\mu_{B}$ and the rise of the strangeness saturation factor $\gamma_{s}$ when going from $\sqrt{s}=17 \mathrm{AGeV}$ to $130 \mathrm{AGeV}$. It would appear that $T$ remains essentially constant for $\sqrt{s}=17 \mathrm{AGeV}$, while at $130 \mathrm{AGeV}$ it rises with $N_{\text {part }}$. The parameter $\mu_{B}$ is fairly independent of the centrality, while the strangeness saturation factor increases with centrality. Despite the rather limited set of analyzed hadron species, the extracted thermal parameters describe other hadron yields, which are at our disposal in central collisions, fairly well.
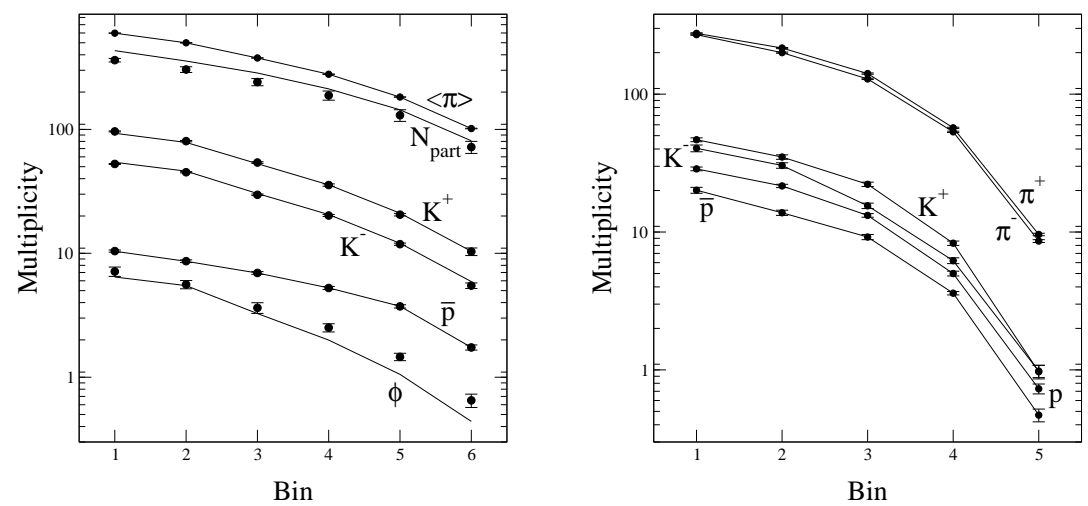

FIG. 1. Comparison of NA49 data (left panel, symbols, [4.5]) and PHENIX data (right panel, symbols, [7) with our model (lines).
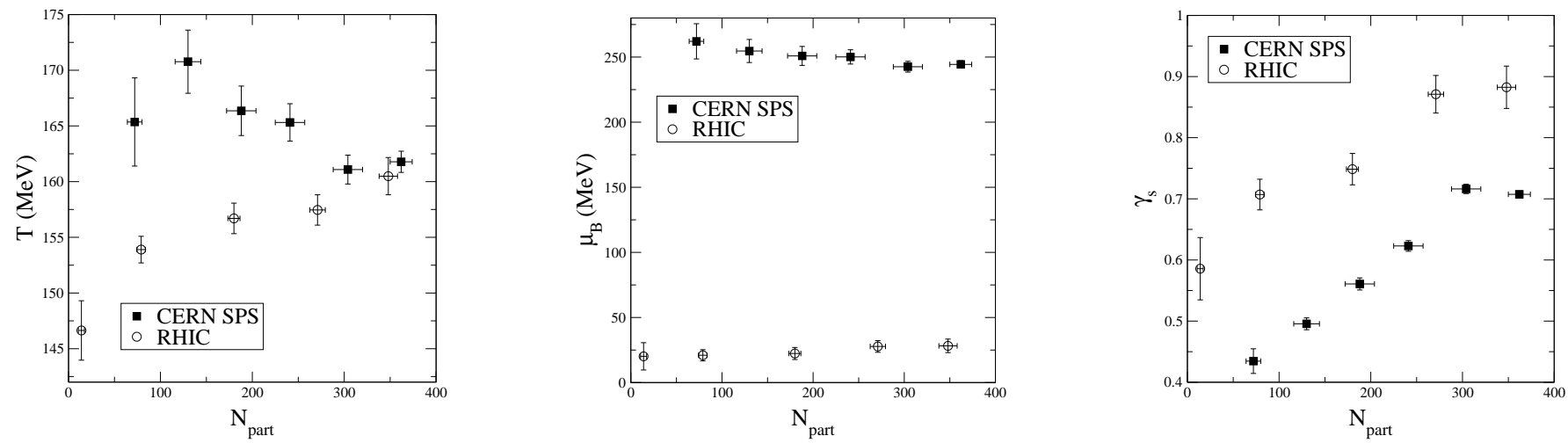

FIG. 2. The temperature, baryo-chemical potential and strangeness saturation factor as a function of $N_{\text {part }}$. Solid squares (open circles) are for the NA49 (PHENIX) data [4.5 ( [7]).

\section{INTERMEDIATE-MASS DILEPTONS}

As pointed out in [9], the dilepton spectra can be analyzed in the same spirit as the hadron multiplicities and hadron momentum spectra, i.e., one discards any detail of the dynamics and asks only for a simple parameterization. As a result, one gets for the thermal dilepton spectrum [9]

$$
\frac{d N}{d^{4} Q}=\frac{5 \alpha^{2}}{36 \pi^{4}} N_{\mathrm{dil}} \exp \left\{-\frac{M_{\perp} \cosh \left(Y-Y_{\mathrm{cms}}\right)}{T_{\mathrm{dil}}}\right\}
$$


where $Q$ is the lepton pair's four-momentum, $M_{\perp}$ its transverse mass and $Y$ its rapidity; $Y_{\text {cms }}$ denotes the fireball rapidity, and $N_{\text {dil }}$ is a normalization factor characterizing the space-time volume of the fireball; flow effects are negligible for invariant mass spectra. Eq. (2) is based on the quark-hadron duality [10].

In 19,11] we have shown that the space-time averaged temperature parameter $T_{\text {dil }} \approx 170 \mathrm{MeV}$ (i.e., a value coinciding with the chemical freeze-out temperature) provides a common description of the low-mass CERES data [12] and the

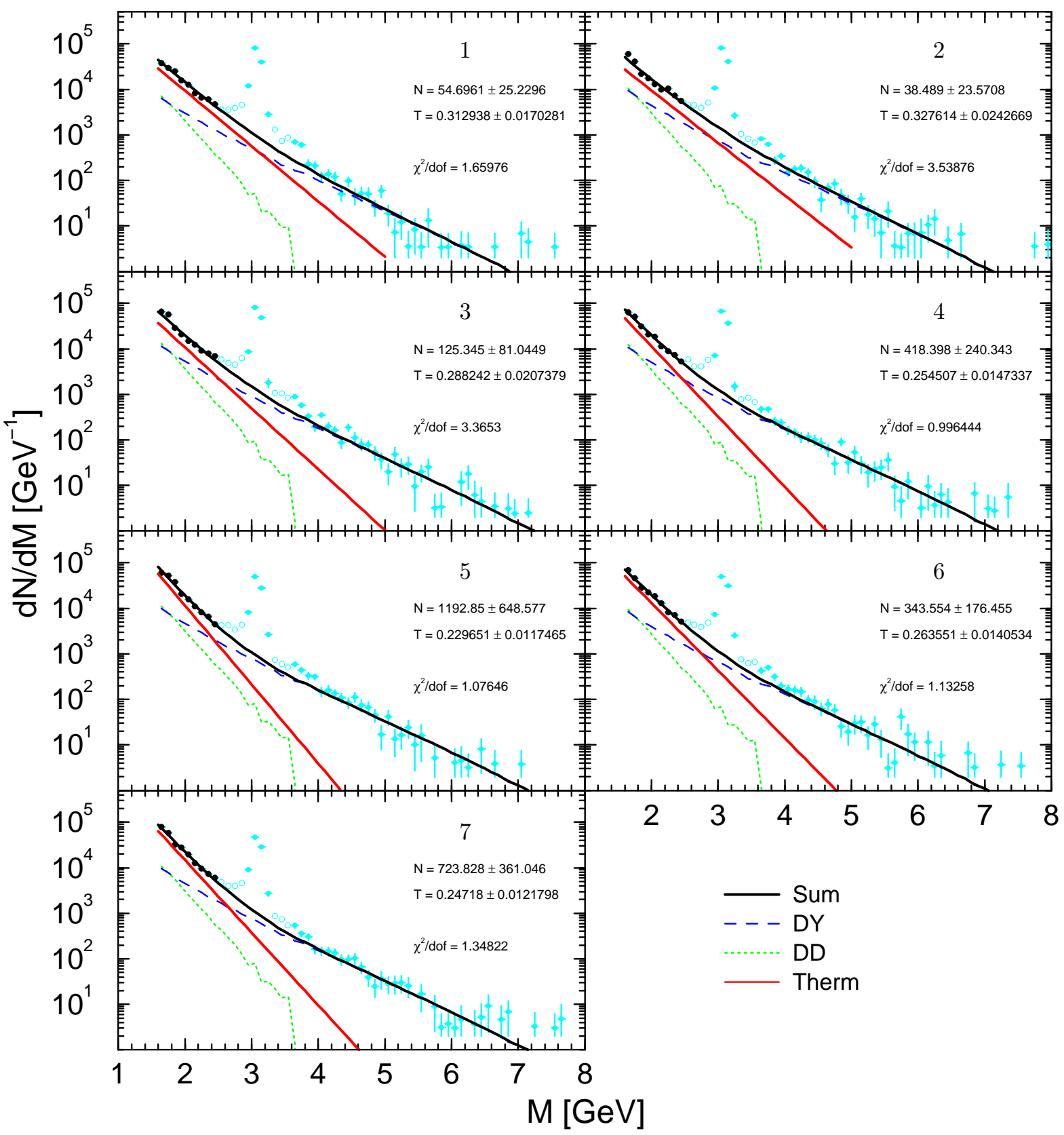

FIG. 3. Fits of the thermal contribution according to Eq. (2) with free temperature parameter and normalization factor to the NA50 data [15]. The data are for 7 centrality bins $\left(E_{T}=19,36,52,67,80,93,110 \mathrm{GeV}\right.$ for bin $\left.1-7\right)$. The Drell-Yan yield and the open charm contributions are calculated as described in detail in [9,11]. 
intermediate-mass NA50 data [13] at $\sqrt{s}=17 \mathrm{AGeV}$; also the WA98 photon data [14] are consistent with this value. It was, therefore, a surprise to us that our analysis of the efficiency corrected and centrality binned NA50 data 15 separately with $N_{\text {dil }}$ and $T_{\text {dil }}$ as free parameters give as optimum fit a temperature scale in the order of $250 \mathrm{MeV}$ [1], see Figs. 3 and 4. At this temperature the spectral shapes of thermal dileptons (Therm) and dileptons from semileptonic correlated decays of open charm mesons (DD) are nearly identical within the NA50 acceptance.

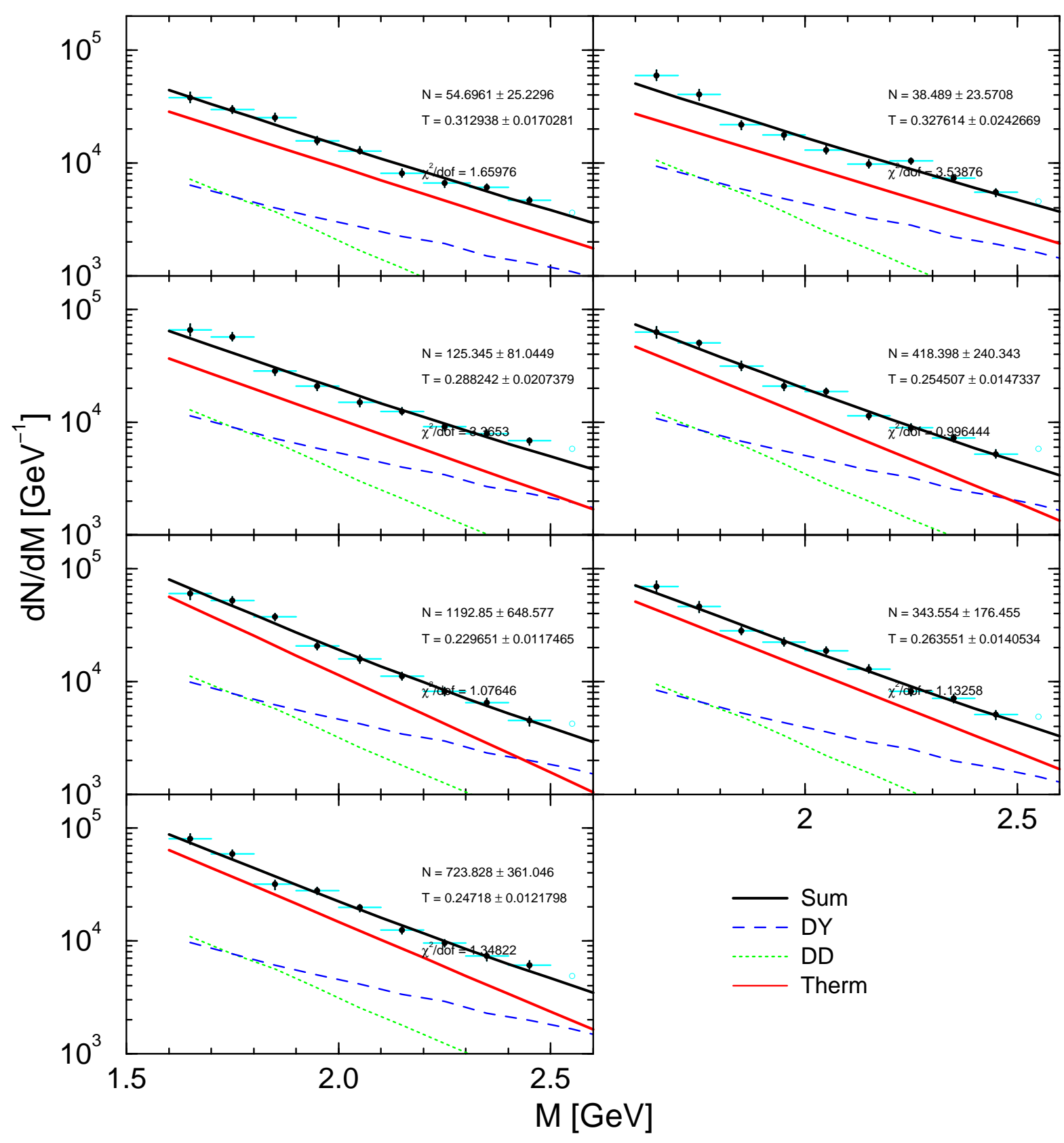

FIG. 4. As Fig. 3 but exhibiting only the intermediate-mass interval below $J / \psi$.

\footnotetext{
${ }^{1}$ Note that in Figs. 3 and 4 the used normalization of both the Drell-Yan yield and the open charm contribution differs by a factor $1 / 1.5$ from the subsequent figures. This covers the uncertainty in fitting the large- $M$ Drell-Yan tail and the relative normalization of the charm contribution.
} 
To illustrate the sensitivity on the temperature parameter we exhibit in Figs. 5 and 6 a comparison with the data when using a fixed value of $T_{\text {dil }}=170 \mathrm{MeV}$. In contrast to the fits displayed in Figs. 3 and 4 the normalization factor now systematically increases with centrality, as expected. This could be interpreted as a hint that the complete disentangling of temperature parameter and normalization factor is not fully adequate.2 Rather, due to the space-time evolution both quantities are correlated. Because of uncertainties in the normalization and the shape of the Drell-Yan (DY) and charm contributions the previously found value of $T_{\text {dil }} \approx 170 \mathrm{MeV}$ [9,11] can not be excluded.

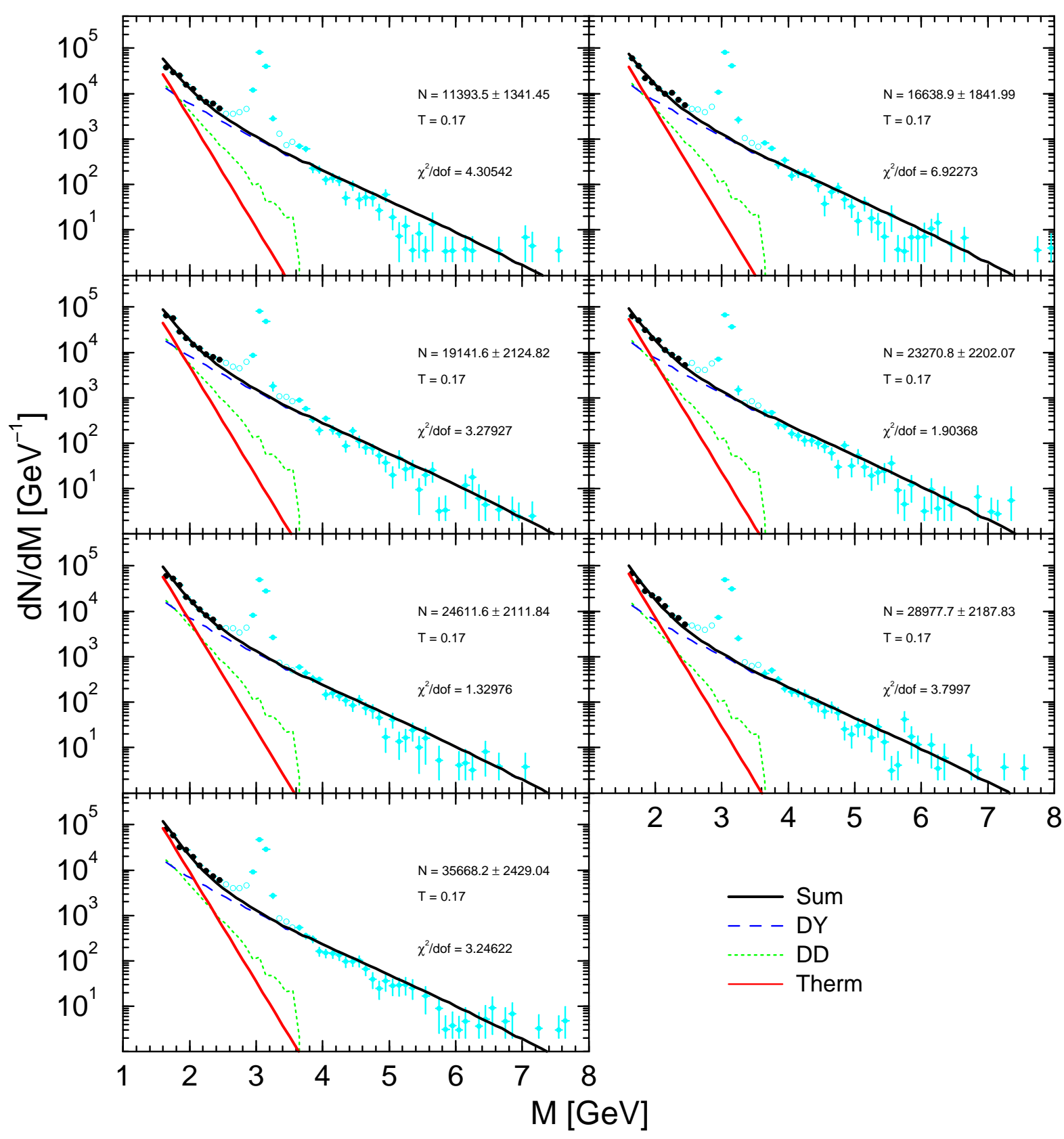

FIG. 5. As in Fig. 3 but for fixed $T=170 \mathrm{MeV}$ and free parameter $N_{\mathrm{dil}}$.

\footnotetext{
${ }^{2}$ We thank R. Rapp for pointing out this possibility.
} 


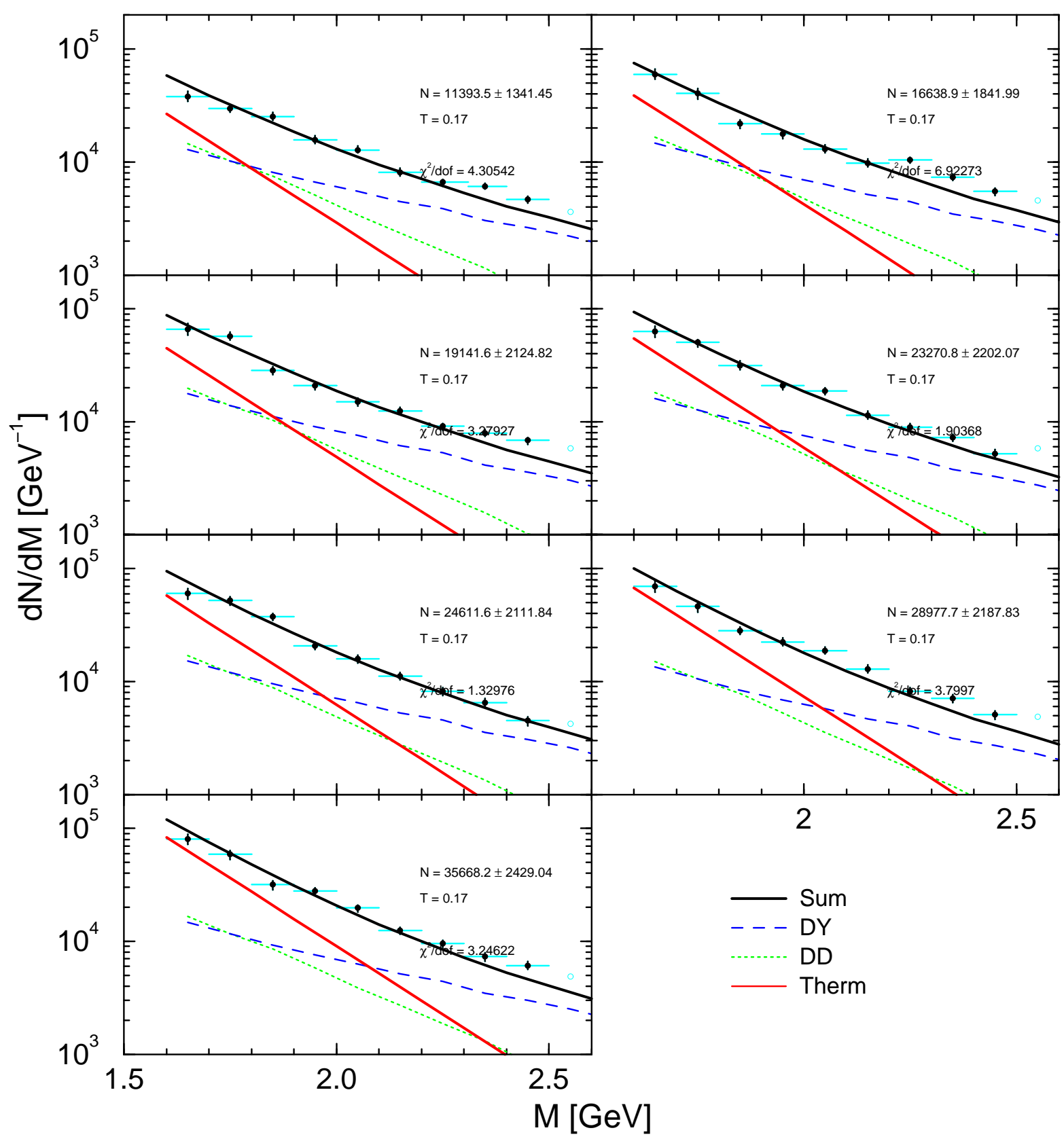

FIG. 6. As in Fig. 1 but for fixed $T_{\text {dil }}=170 \mathrm{MeV}$ and free parameter $N_{\text {dil }}$.

In addition to the invariant mass spectra also transverse momentum spectra integrated over the intermediatemass region $M=1.6 \cdots 2.5 \mathrm{GeV}$ are at our disposal. At first glance, they do not better constrain the temperature parameter $T_{\text {dil }}$ since the large- $Q_{\perp}$ region is dominated by the Drell-Yan yield, see Figs. 7 and 8. The low- $Q_{\perp}$ region, however, favors a smaller value of $T_{\mathrm{dil}}$, while the large- $Q_{\perp}$ region could be fine tuned by a slight change of the intrinsic transverse parton momentum (we use here $\sqrt{\left\langle k_{\perp}^{2}\right\rangle}=0.8 \mathrm{GeV}$ [9]).

In contrast to the invariant mass spectra, where the thermal yield at $T_{\mathrm{dil}} \approx 250 \mathrm{MeV}$ has the same shape as the charm yield, the transverse mass spectra of charm and thermal contributions look similar at $T_{\text {dil }} \approx 170 \mathrm{MeV}$. 


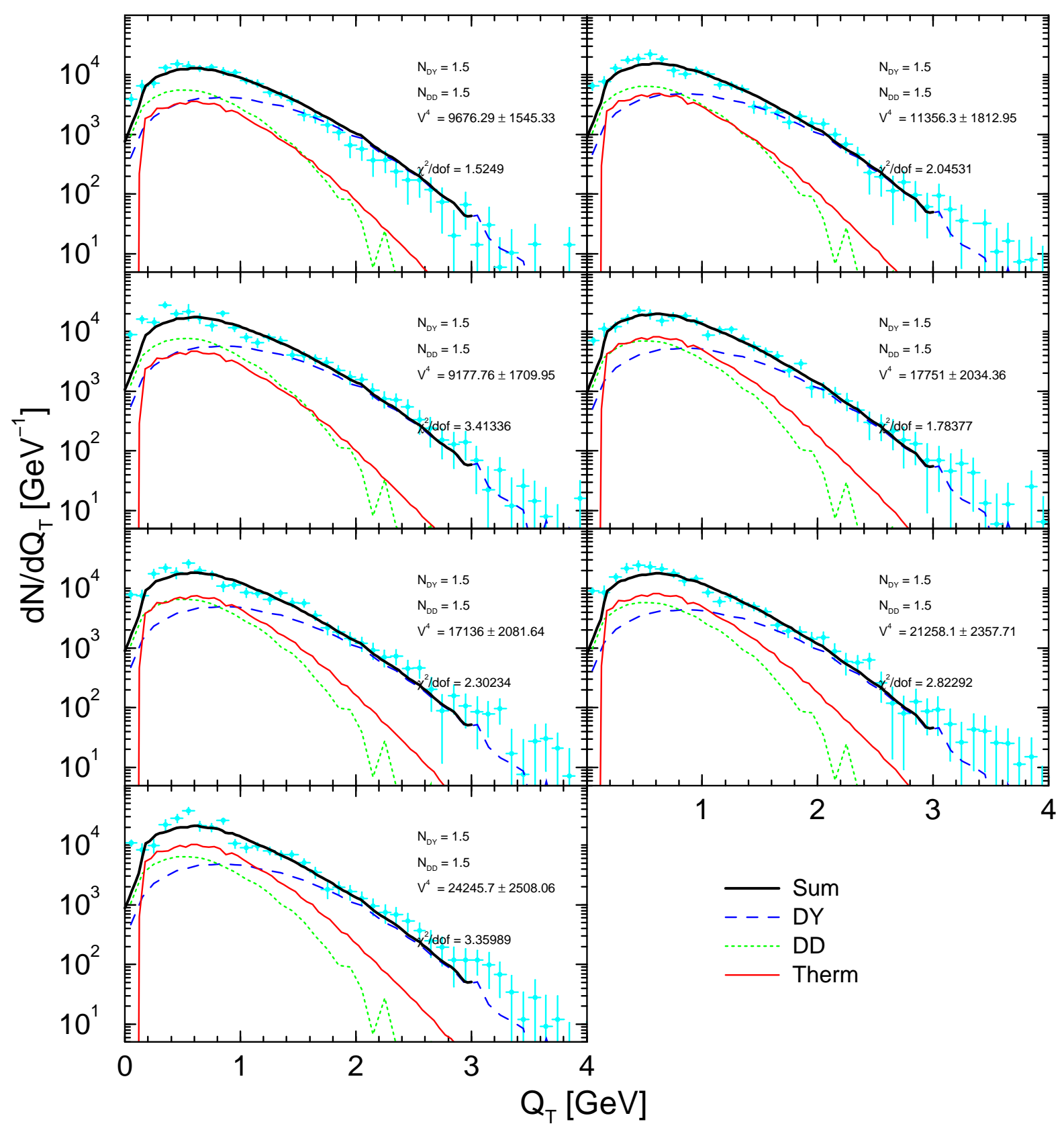

FIG. 7. Transverse momentum spectra for $M$ integrated from $1.6 \cdots 2.5 \mathrm{GeV}$ 15 and for $T_{\text {dil }}=170 \mathrm{MeV}$.

\section{SUMMARY}

The analysis of hadron multiplicities indicates a centrality independence of the chemical freeze-out temperature and baryo-chemical potential for $\sqrt{s}=17 \mathrm{AGeV}$, while at $\sqrt{s}=130 \mathrm{AGeV}$ the baryo-chemical potential is constant but the temperature increases with centrality. The strangeness saturation increases with centrality for both $\sqrt{s}=17$ $\mathrm{AGeV}$ and $130 \mathrm{AGeV}$ and appears to approach unity with further increasing energy and maximum centrality. 


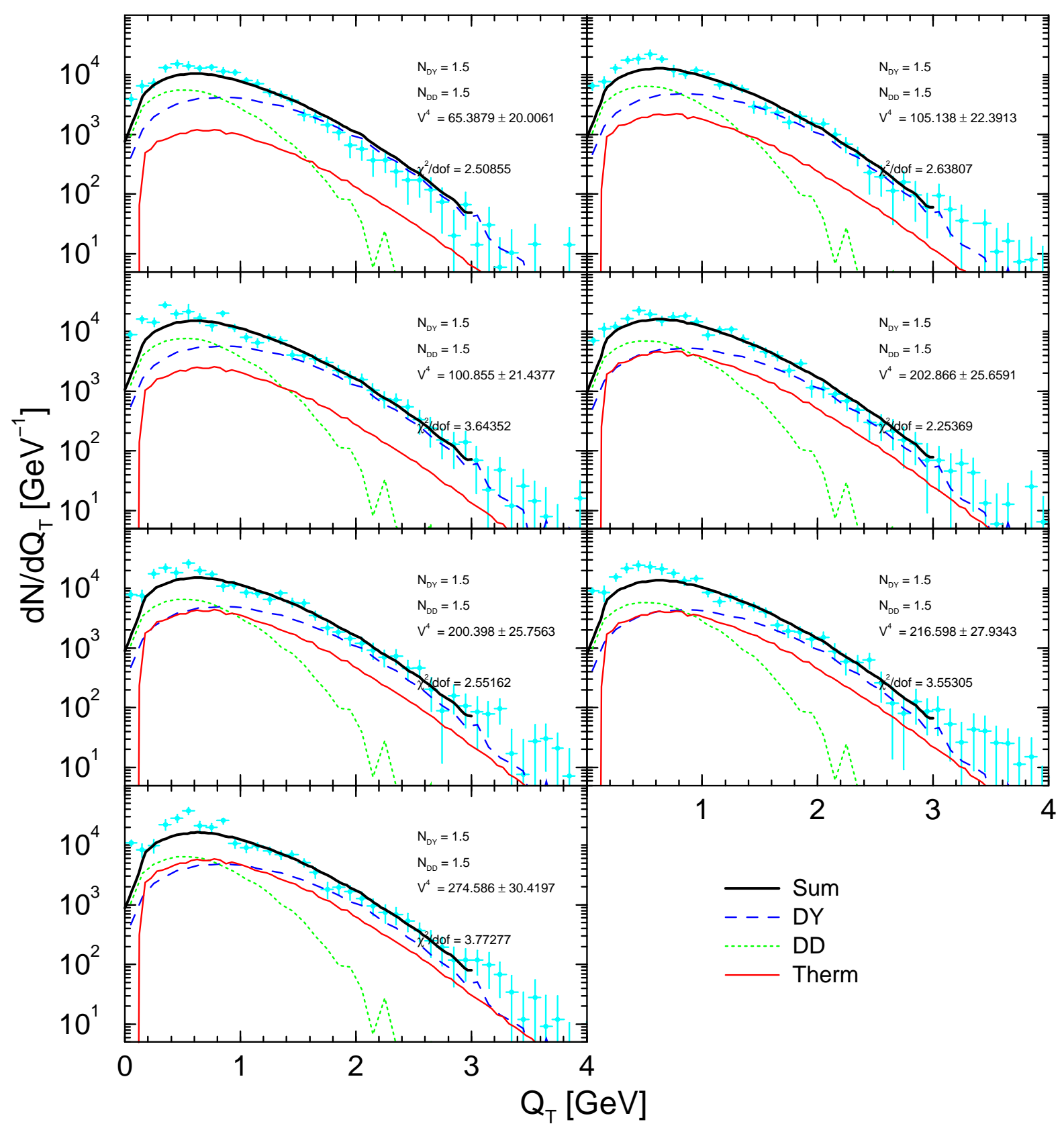

FIG. 8. As Fig. 7 but for $T_{\text {dil }}=250 \mathrm{MeV} . \chi^{2} /$ d.o.f. is marginally larger than for $T_{\text {dil }}=170 \mathrm{MeV}$ in Fig. 7 .

The analysis of the intermediate-mass dilepton spectra taken by the NA50 collaboration is not yet conclusive. Our parameterization of the thermal contribution is based on quark-hadron duality where the shape and the normalization are not interrelated. We find an indication that the space-time averaged temperature determining the shape of dilepton spectra stays fairly independent of centrality, but is significantly larger than previously anticipated in [9, 11] thus being in agreement with values deduced in [16] from photon data. It should be noted, however, that the analysis is sensitive to details of the Drell-Yan and open charm dilepton yields, both their shape and normalization. In view of these inherent uncertainties, the centrality independent value of $T_{\text {dil }} \approx 170 \mathrm{MeV}$ appears still compatible with the NA50 data. 


\section{ACKNOWLEDGMENT(S)}

We are grateful to M. Gazdzicki, F. Sikler, Ch. Blume, and C. Lourenco for discussions on the experimental data and K. Redlich for continuous discussions concerning the thermal model. The work is supported by BMBF $06 \mathrm{DR} 921$.

[1] B. Kämpfer, J. Cleymans, K. Gallmeister, S. Wheaton, hep-ph/0202134, Proc. of the International Workshop XXX on Gross Properties of Nuclei and Nuclear Excitations, Hirschegg, Austria, January 13 - 19, 2002, (Eds.) M. Buballa, W. Nörenberg, B.-J. Schaefer, J. Wambach, p. 158

[2] P. Braun-Munzinger et al., Phys. Lett. B344 (1995) 43, B365 (1996) 1, B465 (1999) 15, B518 (2001) 415

J. Cleymans, K. Redlich, Phys. Rev. Lett. 81 (1998) 5284

[3] F. Becattini, J. Cleymans, A. Keranen, E. Suhonen, K. Redlich, Phys. Rev. C64 (2001) 024901

[4] F. Sikler (NA49 collaboration), Nucl. Phys. A661 (1999) 45c

[5] V. Friese (NA49 collaboration), Nucl. Phys. A698 (2002) 487c

[6] J. Cleymans, B. Kämpfer, S. Wheaton, Phys. Rev. C65 (2002) 027901

[7] K. Adcox et al. (PHENIX collaboration), nucl-ex/0112006

[8] J. Cleymans, B. Kämpfer, S.M. Wheaton, in preparation

[9] K. Gallmeister, B. Kämpfer, O.P. Pavlenko, C. Gale, Nucl. Phys. A688 (2001) 939, A698 (2002) 424c

[10] R. Rapp, J. Wambach, Adv. Nucl. Phys. 25 (2000) 1

[11] K. Gallmeister, B. Kämpfer, O.P. Pavlenko, Phys. Lett. B473 (2000) 20, Phys. Rev. C62 (2000) 057901

[12] B. Lenkeit (CERES collaboration), Nucl. Phys. A661 (1999) 23c

[13] E. Scomparin ( NA50 collaboration), Nucl. Phys. A610 (1996) 331, J. Phys. G25 (1999) 235c

[14] M.M. Aggarwal et al. (WA98 collaboration), Phys. Rev. Lett. 85 (2000) 3595

[15] L. Capelli (NA50 collaboration), Ph. D. thesis, University of Lyon, 2000, Nucl. Phys. A698 (2002) 539c

[16] D.K. Srivastava, B. Sinha, I. Kvasnikova, C. Gale, Nucl. Phys. A698 (2002) 432c

D.K. Srivastava, B. Sinha, Phys. Rev. C64 (2001) 034902 